\title{
Temozolomid über mehr als sechs Zyklen - besser werden die Daten nicht
}

Fragestellung: Gibt es einen Überlebensvorteil für Glioblastompatienten, bei denen die adjuvante Erhaltungschemotherapie mit Temozolomid (TMZ) über mehr als sechs Zyklen fortgesetzt wird?

Hintergrund: Die kombinierte Radiochemotherapie des Glioblastoms sieht sechs adjuvante Zyklen Erhaltungschemotherapie mit TMZ vor [1]. Oft werden jedoch deutlich mehr Zyklen eingesetzt. Retrospektive Analysen, die dieses Vorgehen unterstützen [2], sind dadurch limitiert, dass bei Patienten mit einem frühen Tumorprogress nie sechs oder mehr Zyklen erreicht werden.

Patienten und Methodik: Von 2.002 Patienten innerhalb des deutschen Gliomnetzwerks wurden 142 identifiziert, die mindestens sechs Zyklen adju-

Gramatzki D, Kickingereder P, Hentschel et al. Limited role for extended maintenance temozolomide for newly diagnosed glioblastoma. Neurology 2017; 88: $1422-30$ vante TMZ-Erhaltungschemotherapie ohne Hinweise auf einen Tumorprogress erhielten. Bei 81 Patienten wurde nach sechs Zyklen die Chemotherapie beendet, bei 61 Patienten wurde sie im
Median für insgesamt elf Zyklen fortgesetzt. Beide Gruppen wurden statistisch auf einen Vorteil bei progressionsfreiem beziehungsweise Gesamtüberleben im Log-rank-Test untersucht. Die Analyse von prognostischen Faktoren wie zum Beispiel dem Nachweis einer Isozitratdehydrogenase(IDH)-Mutation oder einer O6-Methylguanin-DNA-Methyltransferase(MGMT)-Promoter-Methylierung erfolgte uni- beziehungsweise multivariat mit der Cox-Regression.

Ergebnisse: Obwohl Patienten mit einer fortgesetzten TMZErhaltungschemotherapie über mindestens sieben Zyklen ein verlängertes progressionsfreies Überleben zeigten (20,5 vs. 17,2 Monate, $\mathrm{p}=0,035)$ fand sich kein Vorteil beim Gesamtüberleben (32,6 vs. 33,2 Monate, $\mathrm{p}=0$,126). Die multivariate Analyse, adjustiert für Alter, Ausmaß der Resektion, Karnofsky-Index, residuellen Tumor, MGMT-Promoter-Methylierung und IDHMutationsstatus, zeigte keine Korrelation von verlängerter TMZ-Erhaltungschemotherapie und progressionsfreiem oder Gesamtüberleben.

Schlussfolgerung: Diese Daten unterstützen nicht die Praxis, die TMZ-Erhaltungschemotherapie über sechs Zyklen hinaus fortzusetzen.

\section{- Kommentar von Martin Uhl, Erlangen}

\section{Keine Daten, die TMZ über mehr als sechs Zyklen unterstützen}

Die vorgestellte Arbeit der Züricher Arbeitsgruppe um Professor Weller ist anderen Untersuchungen überlegen, da sie nur Patienten einschließt, die nach sechs adjuvanten TMZ-Zyklen noch keinen Hinweis auf einen Tumorprogress zeigen. Ältere retrospektive Studien hatten das Problem, dass die Gruppe mit weniger als sechs Zyklen auch Patienten mit einem frühen Tumorprogress enthielten, bei denen die TMZ-Erhaltungschemotherapie abgebrochen wurde. Bereinigt von diesen Patienten zeigt sich nun kein Vorteil der fortgesetzten Erhaltungschemotherapie mit TMZ. Vergleichbare Ergebnisse wurden kürzlich in einem anderen Datensatz von Blumenthal et al. publiziert [3]. Einziger Widerspruch in beiden Studien war lediglich die Frage, ob nicht doch Patienten mit einem methylierten MGMT-Promoter von einer fortgesetzten TMZ-Erhaltungschemotherapie profitieren könnten. Dies bleibt aktuell noch ungeklärt.

Obgleich die hier vorgestellte Studie nicht die höchste Evidenzklasse hat, muss man leider feststellen, dass die Daten nicht besser werden. Um eine endgültige Klärung zu erreichen, bräuchte es eine prospektiv randomisierte Studie, die nach sechs adjuvanten TMZ-Zyklen randomisiert, das heißt zirka neun bis zehn Monate nach der Operation. Angesichts der dafür notwendigen enormen Patientenzahlen, leuchtet es ein, dass es eine solche Studie niemals geben wird. Zusammenfassend kann man sagen, dass wenngleich die Anzahl der adjuvanten TMZ-Zyklen beliebig festgelegt wurde, es weiterhin keine Daten gibt, die ein Fortsetzen der TMZ-Erhaltungschemotherapie über sechs Zyklen hinaus unterstützen.
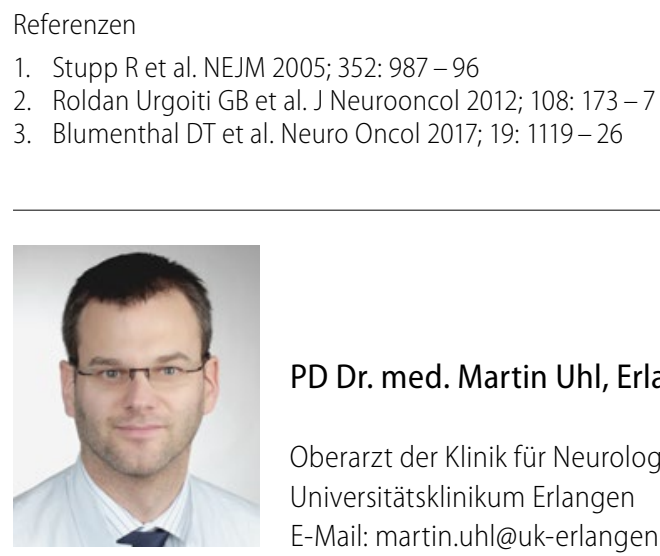

PD Dr. med. Martin Uhl, Erlangen

Oberarzt der Klinik für Neurologie, Universitätsklinikum Erlangen E-Mail:martin.uhl@uk-erlangen.de 